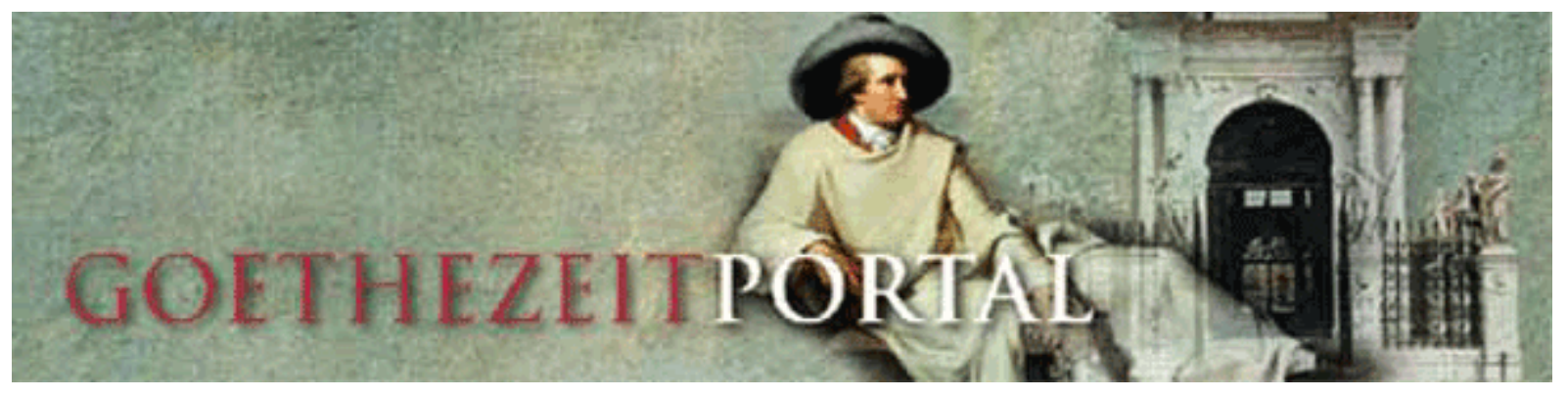

SEBASTIAN DONAT

\title{
Arbeitspensum eines Achtundsiebzigjährigen. \\ Eine unbekannte Agenda Goethes \\ vom November und Dezember 1827
}

\author{
Vorblatt
}

\section{Publikation}

Erstpublikation: Goethejahrbuch 118 (2001), S. 328-345.

Neupublikation im Goethezeitportal

Vorlage: Datei des Autors

URL:

$<$ http://www.goethezeitportal.de/db/wiss/goethe/donat_arbeitspensum.pdf $>$

Eingestellt am 09.08.2004

\section{Autor}

Dr. Sebastian Donat

Ludwig-Maximilians-Universität München

Institut für Allgemeine und Vergleichende Literaturwissenschaft

(Komparatistik)

Schellingstr. 3

80799 München

Emailadresse:<s.donat@1rz.uni-muenchen.de>

\section{Empfohlene Zitierweise}

Beim Zitieren empfehlen wir hinter den Titel das Datum der Einstellung oder des letzten Updates und nach der URL-Angabe das Datum Ihres letzten Besuchs dieser Online-Adresse anzugeben:

Sebastian Donat: Arbeitspensum eines Achtundsiebzigjährigen. Eine unbekannte Agenda Goethes vom November und Dezember 1827 (09.08.2004). In: Goethezeitportal. URL:

$<$ http://www.goethezeitportal.de/db/wiss/goethe/donat_arbeitspensum.pdf $>$ (Datum Ihres letzten Besuches). 
SEBASTIAN DONAT

Arbeitspensum eines Achtundsiebzigjährigen.

Eine unbekannte Agenda Goethes

vom November und Dezember $1827^{1}$

Ich arbeite jeden Tag etwas weg,

aber es giebt des Stoffes so viel,

daß ich denselben sich kaum vermindern sehe. ${ }^{2}$

Das Anlegen von Agenden gehört zu den grundlegenden Elementen der Arbeits- und Lebensorganisation Goethes. Dies bestätigt schon ein kurzer Blick in die bisher vorliegenden Bände der historisch-kritischen Tagebuchedition ${ }^{3}$ sowie in die - freilich bisher noch vollständigere - dritte Abteilung der Weimarer Ausgabe. Zwar wird diesen Arbeitslisten dort erst ab dem Jahr 1802 eine eigene (Teil)Rubrik zugestanden, doch findet sich bereits unter dem 1. Januar 1782 der Tagebucheintrag: „Früh verschiednes in Ordnung. Agenda durchgesehn und überlegt." (GT I,1, S. 129) Im Januar 1790 nahm sich Goethe unter anderem die Fertigstellung der Bearbeitungen von Torquato Tasso, Lila, Jery und Bätely sowie Faust für die Schriften vor; daneben finden sich auf der Agenda aber auch Einträge, wie „Bergwerck“, „Schloß Bau“" oder „Erotica“. (WA III 2, S. 323) Auf Anfang 1799 schließlich datiert eine Arbeitsliste Goethes, die mit dem Nebeneinander von Orts- und Personennamen, Titeln literarischer und naturwissenschaftlicher Werke, Sachbegriffen usw. bereits ein typisches Merkmal der späteren, bis zum März 1832 reichenden Agenden aufweist: „Agenda in Jena | vom 7. Jan. an. | Hiller. | Merseburg. | Farbenlehre | Propylaeen. 2. B. 1 Stück. | Faust. | Mahomet | Uber Piccolomini. | Sammler. | Bibliotheck | Tyger. ${ }^{64}$

Bereits diese frühe Agenda macht deutlich, warum dieser Textsorte bisher wenig Aufmerksamkeit gewidmet wurde. Denn sie enthält zwar fraglos Hinweise auf wichtige und wichtigste Vorhaben Goethes, doch ist dieser, Weizen' mit (im Falle der angeführten Liste sogar recht wenig), Spreu' durchsetzt. Freilich ist diese Einschätzung unserer heutigen Sichtweise auf den vor allem lite-

${ }^{1}$ Die vorliegende Darstellung ist die für den Druck überarbeitete Fassung einer Internetpräsentation, die im Jahre 1999 im Auftrag des Goethe-Instituts erstellt wurde. Siehe Sebastian Donat: Das Goethe-Universum. Eine Agenda vom November und Dezember 1827. URL: http://www.goethe.de/z/11/universum/deindex.htm (23. August 2001). Die Wiedergabe der Handschrift erfolgt mit freundlicher Genehmigung der Russischen Nationalbibliothek St. Petersburg.

2 Brief Goethes an Sulpiz Boisserée vom 27. 2. 1820 (WA IV, 32, S. 174).

3 Johann Wolfgang Goethe: Tagebücher. Historisch-kritische Ausgabe. Hrsg. von Jochen Golz unter Mitarbeit von Wolfgang Albrecht, Andreas Döhler und Edith Zehm. Stuttgart und Weimar 1998ff. Künftig zitiert unter der Sigle GT.

${ }^{4}$ GT II,1, S. 279. Siehe den Kommentar (GT II,2, S. 645). 
rarischen ,Klassiker' verpflichtet und hat wenig mit dem Lebens- und Arbeitsalltag Goethes mitsamt den dazugehörigen Verpflichtungen, Kontakten, großen und kleinen Projekten, Bündelungen und Zerstreuungen der Kräfte zu tun. Es erscheint somit nicht verwunderlich, daß Ferdinand Heitmüller, der Bearbeiter des 13. Bandes der 3. Abteilung der Weimarer Ausgabe, den Abdruck der letzten Agenden Goethes mit Zurückhaltung, ja fast schon entschuldigend, einleitete:

Man wird finden, dass sich in diesen Stücken, trotz der Datirungen, die sie in verschiedene Jahresabschnitte verweisen, mancherlei Wiederholung findet, aber der Gedanke, dass Anschauung in jedem Falle knapper und schon aus diesem Grunde besser als Beschreibung sein möchte, liess die Absicht sie mehr oder weniger zu kassiren nur vorübergehend aufkommen. (WA III 13, S. 237)

Wenn entgegen der skizzierten Praxis nachfolgend eine Agenda Goethes relativ ausführlich vorgestellt und kommentiert werden soll, so geschieht dies vor allem aus zwei Gründen.

Zunächst kann die Agenda vom 1. November und 1. Dezember 1827 allein deswegen Aufmerksamkeit für sich beanspruchen, weil es sich bei ihr um eine bisher unbekannte Handschrift Goethes handelt. Alle damals zugänglichen Arbeitslisten aus dem Zeitraum von 1827-1832 sind in der Weimarer Ausgabe abgedruckt (WA III 13, S. 235-277). Die hier vorgestellte Agenda befindet sich seit 1924 in den Beständen der Russischen Nationalbibliothek in Sankt Petersburg. ${ }^{6}$ Sie paßt in die Lücke zwischen der nach bisherigem Kenntnisstand letzten Agenda von 1827 (datiert auf den 1. September) und der Arbeitsliste von Anfang Juli 1828 (WA III 13, S. 241f.).

Vor allem aber wird das Neben- bzw. Untereinander von knapp fünfzig zunächst disparaten Einträgen aus den unterschiedlichsten Arbeits- und Lebensbereichen Goethes hier als Chance gesehen, das Interessenspektrum und Arbeitspensum des Achtundsiebzigjährigen für den Zeitraum von nur zwei Monaten simultan präsent zu halten. Es reicht von der Pflege privater Kontakte bis zu entoptischen Experimenten, von alltäglichen Finanzangelegenheiten bis zur Mineralogie, vom aktuellen Gesellschaftsleben bis zur redaktionellen Betreuung der Ausgabe letzter Hand. Ein beträchtlicher Teil der Einträge steht dabei in direktem oder indirektem Zusammenhang mit dem letzten von Goethe herausgegebenen Heft (Bd. 6, H. 2, erschienen 1828) seiner Zeitschrift Ueber Kunst und Alterthum. Was diese Agenda nicht zuletzt veranschaulicht, ist die Orientierung gerade des späten Goethe auf das politische, wissenschaftliche

${ }^{5}$ Auch in der neuen historisch-kritischen Edition der Tagebücher Goethes gehören die Agenden nur zu den peripheren Texten. Sie sollen zusammen mit anderen Schriftstücken, „die nur in einem weiteren Sinne dem Tagebuchcorpus zuzurechnen sind oder die sich keiner anderen Werkgruppe zuordnen lassen“, im abschließenden Supplementband wiedergegeben werden (Jochen Golz: Die Edition von Goethes Tagebüchern. Geschichte und Aufgaben. In: GT I,1, S. V-XVI, hier: S. X).

${ }^{6}$ F. 781 (Archiv Tolstoj I. I.), № 1503. 
und literarische Tagesgeschehen und seinen Versuch, den „freyen geistigen Handelsverkehr'“7 nach Kräften zu befördern.

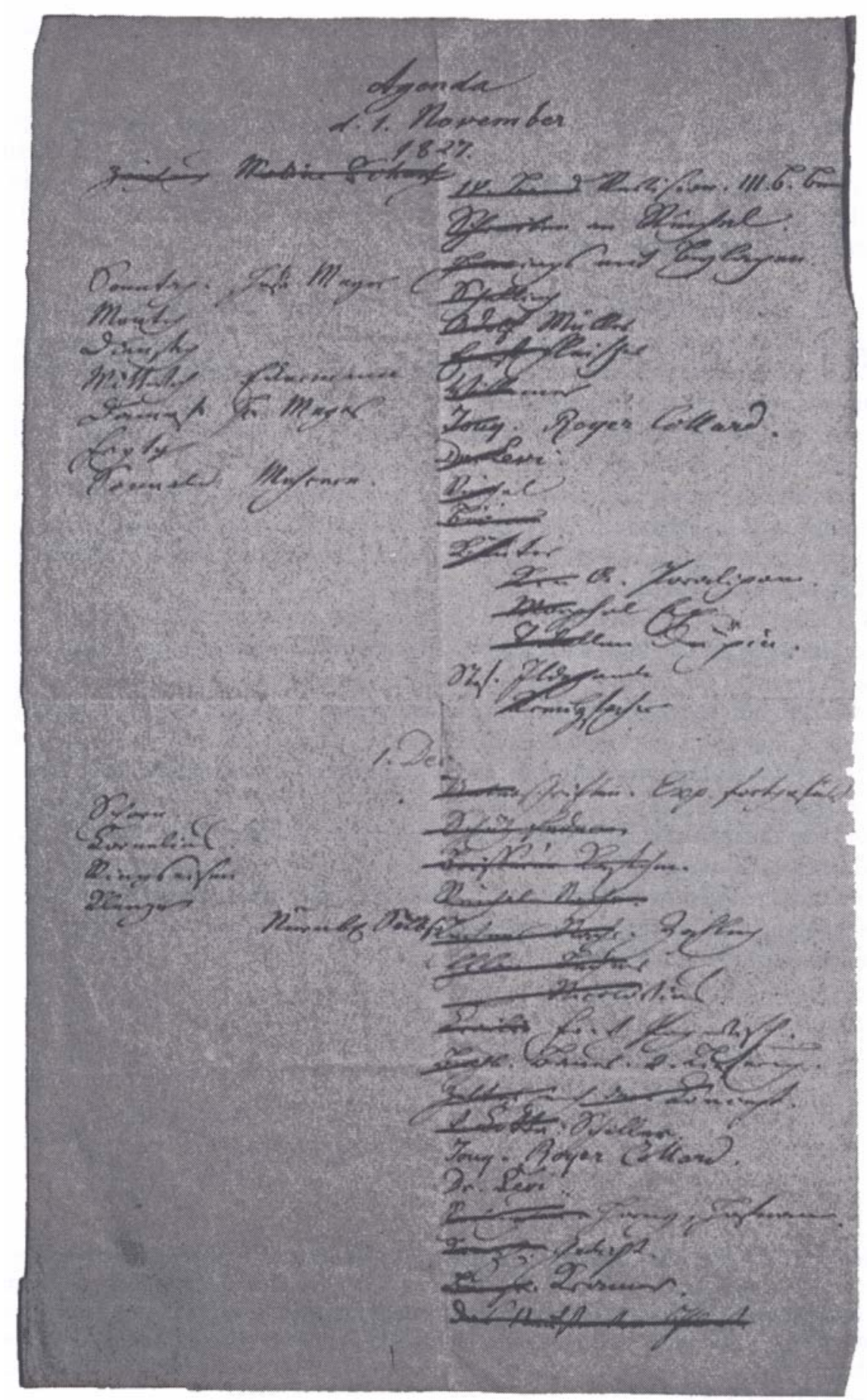

Abb. 1

Johann Wolfgang Goethe: Agenda d. 1. November/1. Dec. 1827, Russische Nationalbibliothek St. Petersburg (Vorderseite)

7 Vorwort zu Carlyles „Leben Schillers“ (FA I 22, S. 869-883, hier: S. 870). 


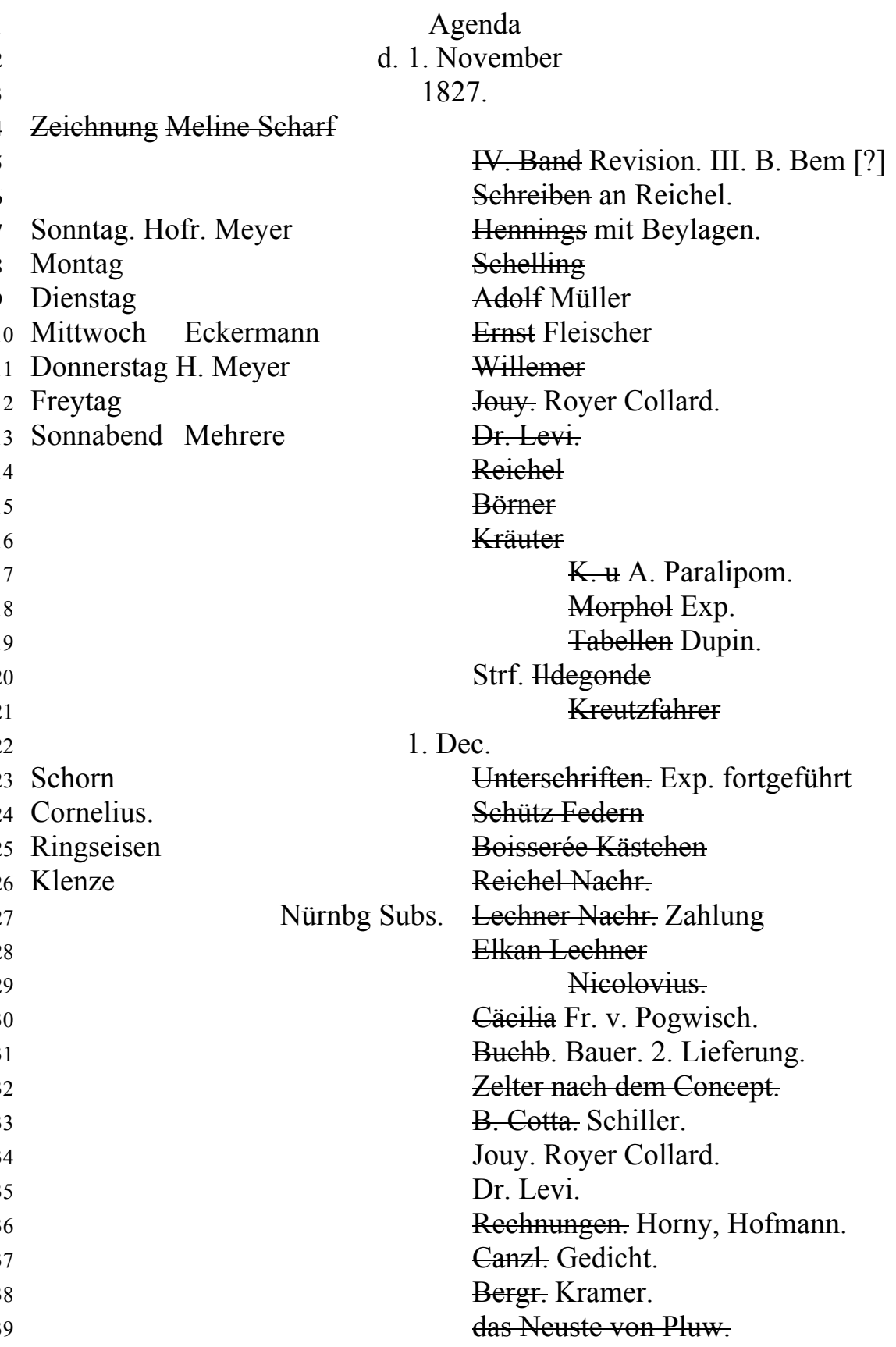


Bei der Agenda handelt es sich um ein doppelt gefaltetes Blatt grobes Konzeptpapier im Folioformat. Die Notizen sind durchgehend von Goethes Hand, dabei überwiegend in roter (Z. 7-13 1. sowie - mit wenigen Ausnahmen - Z. 22-39), zum Teil auch in schwarzer Tinte (Z. 1-3, 5-21 r. sowie einzelne weitere Notizen). Durchgestrichene Einträge sind im Autograph als erledigt abgestrichen. Auf der Rückseite findet sich von fremder Hand in schwarzer Tinte die Notiz „Göthes Handschrift, erhalten [ein nicht entzifferbares Wort; S.D.] zu Weimar Ao 33 “. Wohl auf die Anlage der Agenda bezieht sich der Tagebucheintrag Goethes vom 1. November 1827: „Sendungen vorbereitet. Einiges geordnet und notirt, was zunächst zu leisten sey“ (WA III 11, S. 131).

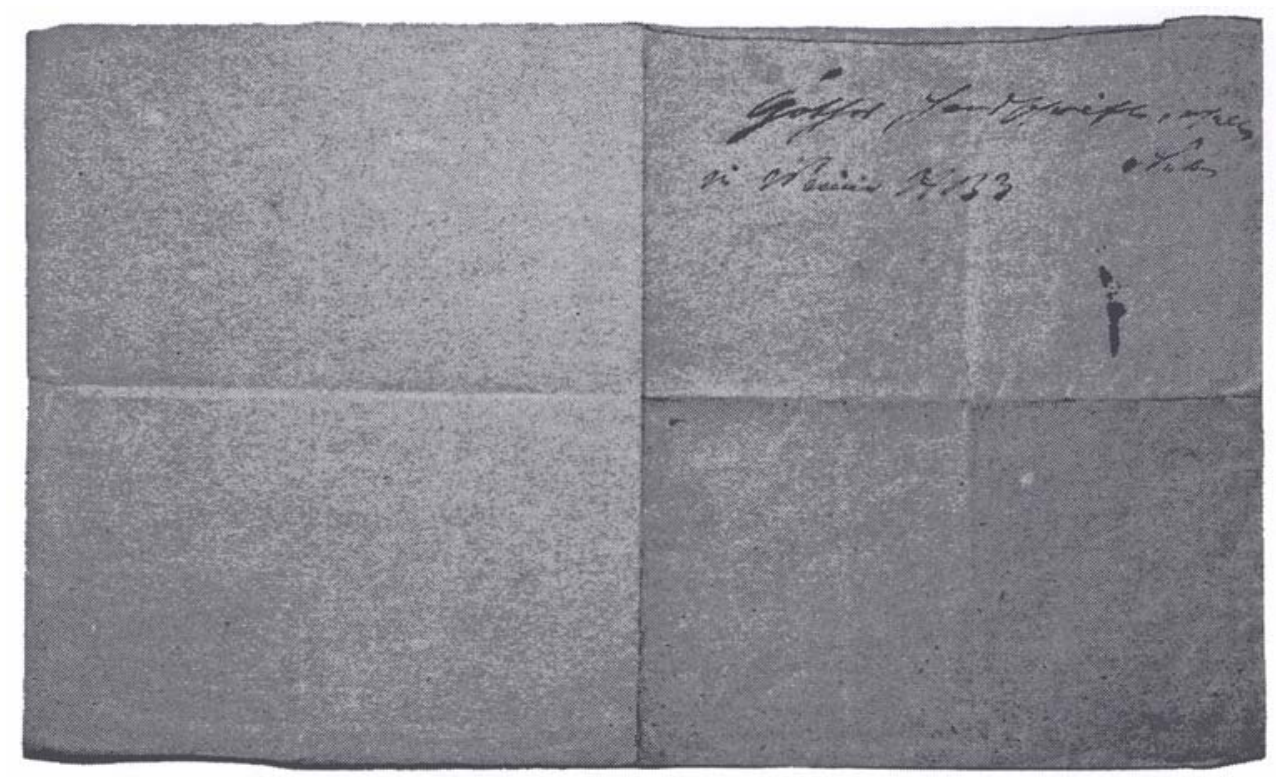

Abb. 2

Johann Wolfgang Goethe: Agenda d. 1. November / 1. Dec. 1827, Russische Nationalbibliothek St. Petersburg (Rückseite - im Original ein Blatt)

Kommentar:

Z. 1: Agenda - ,was zu tun, zu besorgen, zu erledigen ist; mehrf[ach] zugl[eich] für dessen schriftl[iche] Fixierung". 8

Z. 4: Zeichnung Meline Scharf - Der Eintrag Goethes bezieht sich wahrscheinlich auf Amalie (Meline) Henriette Scharff, geb. Willemer (1788-1872), Tochter Johann Jakob Willemers aus erster Ehe, verheiratet mit Friedrich Scharff, Kaufmann in Frankfurt am Main. Über die schwere Krankheit ihrer Tochter Röschen (1806-1841) hatte Marianne v. Willemer Goethe in ihrem Brief vom 9. Dezember 1827 berichtet und für sie um die Übersendung einer Mainansicht

${ }^{8}$ Goethe-Wörterbuch. Hrsg. von der Akademie der Wissenschaften der DDR, der Akademie der Wissenschaften in Göttingen und der Heidelberger Akademie der Wissenschaften. Stuttgart usw. 1978 ff., Bd. 1, Sp. 285. 
mit handschriftlicher Widmung gebeten. ${ }^{9}$ Tatsächlich legte Goethe in seiner Antwort vom 3. Januar $1828^{10}$ zwar nicht die gewünschte Zeichnung von Rosette Städel bei, dafür jedoch Darstellungen von seiner Stadtwohnung und seinem Gartenhaus mit den Versen „Warum stehen sie davor?...“ und „Übermüthig sieht's nicht aus... “11. Er kommentierte die Beilagen folgendermaßen:

Ihrem neulich ausgesprochenen Wunsche, theuerste Freundin, kann ich leider nicht entgegen kommen, denn die Platte von jenen angenehmen Bildchen hat sich verloren, kein Abdruck ist mehr vorhanden; doch kann ich meine Bereitwilligkeit durch ein paar andere Aussichten mit Vergnügen beweisen, die freylich keinen freyen Fluß, keine bedeutende Stadt darzustellen hatten, vielmehr von Einfalt und Beschränkung das bescheidenste Zeugniß geben; vielleicht aber kann abgesonderte Ländlichkeit und gemäßigt-städtisches Wesen nicht besser ausgedruckt werden. Auch sehen Sie einige Reimzeilen von meiner Hand darunter geschrieben. Und so wird denn wohl dem guten Kinde, dem Sie jenes Christgeschenk zudachten, durch Gegenwärtiges zum neuen Jahr noch einige Freude. (WA IV 43, S. 226)

Z. 5: $\quad$ IV. Band Revision. III. B. Bem [?] - Die Notiz bezieht sich auf die Vollständige Ausgabe letzter Hand - hier: die Oktavausgabe (C) - von Goethes Werken, die ab 1827 bei Cotta in Stuttgart und Tübingen erschien. In seinem Brief an Carl Wilhelm Göttling, der an der Redaktion der Ausgabe letzter Hand beteiligt war, berichtete Goethe am 17. November 1827 über den Abschluß der Arbeiten am 4. Band: „Die Octavausgabe schreitet vor, die Correcturen zum vierten [Bande] sind abgegangen." (WA IV 43, S. 174)

Z. 6: $\quad$ Schreiben an Reichel - Dieser Eintrag steht im Zusammenhang mit Goethes Brief vom 24. November 1827 an Wilhelm Reichel (1783 - nach 1836), den Faktor und Korrektor der Augsburger Druckerei von Goethes Verleger Cotta, der sich ebenfalls auf die Ausgabe letzter Hand bezieht (WA IV 43, S. 180f.).

Z. 7 li.: Sonntag. Hofr. Meyer - Ein Besuch Johann Heinrich Meyers (1760-1832) bei Goethe an einem Sonntag ist in den Tagebuchaufzeichnungen vom November 1827 erst für den 25. November vermerkt (WA III 11, S. 141). Wahrscheinlich bezieht sich die Notiz jedoch auf Anfang November. So sind für die Woche vom Sonntag, den 4. November bis zum Sonnabend, den 10. November 1827 die weiteren auf der Agenda erwähnten Besuche Eckermanns am Mittwoch (7. November, WA III 11, S. 134) und Meyers am Donnerstag (8. November; ebd.) im Tagebuch Goethes eingetragen. Die Einladung Meyers für Sonntag, den 4. November wird geplant gewesen sein, kam jedoch offenbar nicht zustande.

Z. 7 re.: Hennings mit Beylagen - Die Notiz bezieht sich auf Goethes Brief vom 27. November 1827 an den Juristen, Philosophen und Physiker Leopold Dorotheus

9 Siehe die entsprechende Passage aus dem Brief Marianne v. Willemers an Goethe vom 9. 12. 1827: „Die Tochter meiner guten Meline (Scharff), die nun schon 3 Jahre lang das Bett nicht verlassen konnte [...], würde es sehr glücklich machen, wenn Sie vielleicht noch eine jener kleinen Mainansichten entbehren und wohl gar mit ein paar Worten verschönern mögen; ich bin überzeugt, das Christkindchen würde ihr nichts Lieberes bringen." (Marianne und Johann Jakob Willemer. Briefwechsel mit Goethe. Dokumente. Lebens-Chronik. Erläuterungen. Hrsg. von Hans-J. Weitz. Frankfurt a. M. 1965, S. 192.)

${ }^{10}$ Willemer-Briefwechsel (Anm. 9), S. 192f., sowie WA IV 43, S. 226-228.

11 FA I 2, S. 864 und 526. Die Stiche sind wiedergegeben in Goethes Leben in Bilddokumenten. Hrsg. von Jörn Göres. München 1981 (Repr. Augsburg 1999), S. 146f., Abb. 234 und 236. 
von Henning gen. von Schönhoff (1791-1866), ab 1825 Philosophieprofessor in Berlin. Henning, Goethes ,chromatischer Freund, ${ }^{12}$ gehörte zu den wenigen etablierten zeitgenössischen Akademikern, die Goethes Farbenlehre nicht nur akzeptierten, sondern sogar förderten und verbreiteten. Wohl während seines Besuchs in Weimar im September 1827 hatte Henning Goethe auf eine neue entoptische Versuchsanordnung aufmerksam gemacht. Am 24. November 1827 notierte Goethe in seinem Tagebuch: „Promemoria an Herrn von Hennings auf entoptische Versuche bezüglich" (WA III 11, S. 140). Er beschrieb in seinem Brief an Henning (WA IV 43, S. 190-193) ausführlich seine erfolgreiche Durchführung dieses Experiments und regte den Adressaten dazu an, den Versuch selbst nachzuvollziehen. Dazu diente nicht zuletzt die auf der Agenda erwähnte „Beylage“: eine Skizze der Versuchsanordnung. ${ }^{13}$

Z. 8 li.: Montag - Vgl. den Kommentar zu Z. 7 li.

Z. 8 re.: Schelling - Nach zwölfjähriger Pause hat Goethe dem 1827 von König Ludwig I. als Professor nach München berufenen Friedrich Wilhelm Joseph Schelling (1775-1854) am 26. Oktober 1827 geschrieben. ${ }^{14}$ Kanzler Müller, der sich zu dieser Zeit in München aufhielt, berichtet in seiner Tagebuchaufzeichnung vom 31. Oktober 1827 über Schellings Reaktion auf diesen Brief:

Bey Schelling war eben ein Brief von Göthe [...] eingetroffen, der grose Freude machte. Ich mußte den ganzen Hergang des 28. Aug. erzählen [Ludwig I. hatte überraschend an der Feier zu Goethes 78. Geburtstag in Weimar teilgenommen; S.D.] und versprechen, mein Gedicht zu senden. Schelling hegt grose Hoffnungen vom König, traut ihm tiefe Plane und Combinationen, grose Vestigkeit zu. „Goethe ist seit dem 28. Aug. nun auch der unsrige, Mitgenosse, Mitverbündeter der ganzen neuen Periode rühmlicher Bestrebungen.“ Der König wollte ihm gleichsam im voraus Mitgenuß der Zukunft geben. Andren Theils habe der König der Welt durch diese Reiße Pfand und Bürgschaft seiner ächten Gesinnung gegeben. ${ }^{15}$

Z. 9 li.: Dienstag - Vgl. den Kommentar zu Z. 7 li.

Z. 9 re.: Adolf Müller - Bezug nicht ermittelt.

Z. 10 li.: Mittwoch Eckermann - Besuche von Johann Peter Eckermann (1792-1854) sind in Goethes Tagebuch vermerkt für die ersten drei Mittwoche im November (7., 14. und 21. November $1827^{16}$ ). Vgl. den Kommentar zu Z. 7 li.

Z. 10 re.: Ernst Fleischer - Ernst Gerhard Fleischer (1799-1832), Verleger und Buchhändler in Leipzig, besuchte Goethe am 11. Oktober 1827 und überreichte ihm ein Widmungsexemplar des Parnasso Italiano (hrsg. von Gottlob Heinrich Adolph Wagner, Leipzig 1826). Carl Victor Meyer, damals ebenfalls bei Goethe zu Gast, beschreibt dessen Reaktion darauf:

Am Donnerstag abend [11. Oktober] ließ sich der Buchhändler Fleischer aus Leipzig anmelden und überreichte ihm beim Abschiede ein schönes Kästchen, worin sich, zierlich in Maroquin gebunden, seine neueste Ausgabe der italienischen Klassiker befand, mit folgender Deducation:

\footnotetext{
12 Siehe Goethes Tagebuch vom 21. 8. 1828 (WA III 11, S. 266f.).

Wiedergegeben in WA IV 43, S. 387.

14 WA IV 43, S. 125f.; vgl. den Tagebucheintrag vom 26. 10. 1827 (WA III 11, S. 128).

15 Kanzler von Müller: Unterhaltungen mit Goethe. Kritische Ausgabe. Besorgt von Ernst Grumach. Weimar 1956, S. 171.

16 Siehe WA III 11, S. 134, 136 und 139.
} 
„Al Principe del Poetica“, Goethen, worüber sich der Alte gewaltig freute. ${ }^{17}$

Bereits am folgenden Tag notierte Goethe: „Secretär Kräuter wegen der Fleischerischen Angelegenheiten“ (WA III 11, S. 124). Als Zeichen, „womit ich besser als mit Worten den freudigen Dank ausdrückte, welchen ich bey'm Anblick der herrlichen Gabe empfinde, die Sie mir verleihen wollen" (Brief Goethes an Gottlob Heinrich Adolph Wagner vom 29.10.1827, WA IV 43, S. 138), übersandte Goethe an den Herausgeber und den Verleger des ihm gewidmeten Buches ein „Kästchen mit Kunstarbeiten in Metall Werth 10 Rthlr. an Herrn Adolph Wagner in Leipzig, inliegend ein Futteral mit Bronzemedaillen für Ernst Fleischer in Leipzig“" (Tagebuch, 3.11.1827, WA III 11, S. 132). ${ }^{18}$

Z. 11 li.: Donnerstag H. Meyer - Meyers Besuch bei Goethe ist im Tagebuch vermerkt für Donnerstag, den 1. November 1827 (WA III 11, S. 131), aber auch für die beiden folgenden Donnerstage (WA III 11, S. 134 und 137). Vgl. den Kommentar zu Z. 7 li.

Z. 11 re.: Willemer - Der Eintrag steht wohl im Zusammenhang mit den beiden Briefen Marianne v. Willemers (1784-1860) an Goethe vom 24. November und 9. Dezember 1827. ${ }^{19}$ Goethe beantwortete sie am 3. Januar 1828. Vgl. den Kommentar zu Z. 4.

Z. 12 li.: Freytag - Vgl. den Kommentar zu Z. 7 li.

Z. 12 re.: Jouy. Royer Collard - Die Einträge beziehen sich wohl auf Victor-JosephÉtienne Jouy (1764-1846), französischer Dramatiker, Operndichter, sowie auf Pierre Paul Royer-Collard (1763-1845), französischer Philosoph und Staatsmann.

Goethe besaß Jouys L'Hermite de la chaussée - d'Antin ou Observations sur les moeurs et les usages parisiens au commencement du XIX siècle $^{20}$. Im Gespräch mit dem französischen Historiker, Musik- und Kunstschriftsteller Ludovic Vitet zollte Goethe diesem Werk im April 1827 höchstes Lob. ${ }^{21}{ }_{-}$ Möglicherweise steht der Eintrag auf der Agenda im Zusammenhang mit dem Münchenaufenthalt von Carl Friedrich Zelter im September und Oktober 1827 sowie dessen anschließendem Besuch in Weimar. In seinem Brief an Goethe vom 27.-30. September 1827 berichtete Zelter über eine Münchener Aufführung der Oper Die Vestalin unter der Leitung des Komponisten und Berliner Generalmusikdirektors Gaspare Spontini - das Libretto zu dieser preisgekrönten Oper stammte von Jouy. ${ }^{22}$

Die Erwähnung Royer-Collards bezieht sich wahrscheinlich auf dessen politische Tätigkeit: Von 1815 bis 1839 war er liberaler Abgeordneter der französischen Deputiertenkammer. Die politischen Wirren in Frankreich im Winter 1827/28 (Auflösung der Deputiertenkammer am 5. November 1827, Ausfall der Neuwahlen, Einsetzung eines liberalen Kabinetts durch Karl X. am 4. Janu-

17 Goethes Gespräche. Eine Sammlung zeitgenössischer Berichte aus seinem Umgang. Auf Grund der Ausgabe und des Nachlasses von Flodoard Freiherrn von Biedermann. Ergänzt und hrsg. von Wolfgang Herwig. München 1998, Bd. 3.2, S. 229-231, hier: S. 231, Nr. 6088.

${ }^{18}$ Vgl. Rudolf A. Fleischer: Die Buchhändlerfamilie Fleischer in der Zeit Goethes. Leipzig 1937, S. 115-118.

19 Willemer-Briefwechsel (Anm. 9), S. 189-192.

205 Bde. Paris 1814-15; siehe Goethes Bibliothek. Katalog. Bearbeitet von Hans Ruppert. Weimar 1958 (Repr. Leipzig 1978), S. 226, Nr. 1592.

21 Gespräche (Anm. 17), Bd. 3.2, S. 108, Nr. 5961.

22 Siehe MA 20.1, S. 1054, sowie den Kommentar (MA 20.3, S. 277). 
ar 1828) sind in Goethes Tagebuchaufzeichnungen und Briefen dieser Zeit an vielen Stellen präsent. ${ }^{23}$

Z. 13 li.: Sonnabend. Mehrere - Vgl. den Kommentar zu Z. 7 li. Für Sonnabend, den 10. November 1827, vermerkt Goethes Tagebuch die Besuche von Friedrich Wilhelm Riemer, Carl Vogel und James Henry Lawrence (WA III 11, S. 135).

Z. 13 re.: Dr. Levi - Die Zuordnung dieser Notiz muß offenbleiben. Im Kontext der Briefe und Tagebucheinträge Goethes findet sich keine weitere Erwähnung eines „Dr. Levi““.

Z. 14: Reichel - Vgl. den Kommentar zu Z. 6.

Z. 15: Börner -Vom März 1827 an hat Goethe wiederholt bei dem Leipziger Maler und Kunsthändler Carl Gustav Börner (1790-1855) Graphiken bestellt. Am 30. Oktober 1827 wird im Tagebuch die Durchsicht einer solchen Sendung vermerkt (WA III 11, S. 130), die neben im August bestellten Werken von Giulio Romano, Jakob Philipp Hackert und anderer ${ }^{24}$ „eine Mappe mit Zeichnungen zur Auswahl“" enthielt (WA IV 43, S. 369). Am 8. November 1827 sandte Goethe Börner Aufstellungen über daraus anzukaufende Stücke ${ }^{25}$ und bat am selben Tag den Weimarer Bankier Julius Elkan um die Überweisung des Betrags (WA IV 43, S. 159f.). In seinem Brief an Börner vom 21. November 1827 schickte er die restlichen Zeichnungen an diesen zurück. ${ }^{26}$

Z. 16: Kräuter - Die folgenden drei Einträge (Z. 17-19) stellen möglicherweise Aufträge Goethes an seinen Sekretär Friedrich Theodor Kräuter (1790-1856) dar.

Z. 17: K. u A. Paralipom. - Goethe beschäftigte sich zu dieser Zeit intensiv mit der Vorbereitung des 2. Heftes des 6. Bandes seiner Zeitschrift Ueber Kunst und Alterthum. - Als „Paralipomenon“ (von griech. paraleípein: auslassen) bezeichnete Goethe „einstweilen beiseitegelegte, nicht zum Druck gegebene oder zur Veröffentlichung ungeeignete [Texte], deren eventuelle spätere Publikation er dem Urteil seiner literarischen Nachlaßverwalter überließ.“27 Für das zweite Heft des sechsten Bandes von Ueber Kunst und Alterthum sind mehrere solche Paralipomena überliefert (FA I 22, S. 689-717).

Z. 18: Morphol. Exp. - Die Zuordnung dieser Notiz muß offenbleiben.

Z. 19:Tabellen Dupin - Goethe entlieh die Schrift des französischen Mathematikers, Ingenieurs und Nationalökonomen Pierre Charles François Baron de Dupin (1784-1873) Forces productives et commerciales de la France vom 4. Oktober 1827 bis 18. April 1828 aus der Weimarer Bibliothek. ${ }^{28}$ Seine Lektüre bezeugen die Tagebucheinträge vom 26. September 1827 und - im vorliegenden Kontext einschlägig - 19. November 1827 (WA III 11, S. 115 und 138) sowie Goethes Brief an Dupin vom 12. Oktober 1827 (WA IV 43, S. 105f.). Möglicherweise im Zusammenhang mit den von Goethe auf der Agenda notierten ,Tabellen' steht eine Zusammenkunft in seinem Haus am 16. November 1827, die in Frédéric Sorets Brief an Pierre Dumont vom 10. Dezember 1827 beschrieben wird:

${ }^{23}$ Siehe Goethe an den Grafen Kaspar von Sternberg, 27.11.1827 (WA IV 43, S. 185f.) sowie an Carl Friedrich von Reinhard, 28.1.1828 (WA IV 43, S. 267f.).

${ }^{24}$ Siehe Goethe an Carl Gustav Börner, 17.8. u. 11.9.1827 (WA IV 43, S. 24f. und 57 f.).

${ }^{25}$ Siehe WA IV 43, S. 157f. sowie Tagebuch (WA III 11, S. 134).

${ }^{26}$ Siehe WA IV 43, S. 176 sowie Tagebuch, 20.11.1827 (WA III 11, S. 139).

27 Gero von Wilpert: Goethe-Lexikon. Stuttgart 1998, S. 807. Vgl. Anne Bohnenkamp: Zur Verwendung des Begriffs ,Paralipomena'. In: Dies.: ,... das Hauptgeschäft nicht außer Augen lassend “. Die Paralipomena zu Goethes ,Faust'. Frankfurt/M, Leipzig 1994, S. 57-66.

28 Siehe Goethe als Benutzer der Weimarer Bibliothek. Ein Verzeichnis der von ihm entliehenen Werke. Bearbeitet von Elise von Keudell, hrsg. von Werner Deetjen. Weimar 1931 (Repr. Leipzig 1982), S. 297, Nr. 1872. 
Neulich traf ich ihn [Goethe] damit beschäftigt, die Bevölkerungslisten des Großherzogtums Ort für Ort durchzusehen, und daneben die der Kinder, die eine Schule besuchen; er drückte mir die Feder in die Hand, und ich mußte die Zahlen eintragen; wir fanden Gemeinden, in denen ein Viertel, sogar fast ein Drittel der Einwohnerschaft zur Schule geht, ein Beweis einmal für die ungeheure Bevölkerungszunahme [...], dann auch für das Interesse, das man der Schule entgegenbringt. Als zweites Ergebnis zeigte sich, daß im Großherzogtum SachsenWeimar, ganz im Gegensatz zu der Feststellung Dupins [in seiner Karte vom Kulturzustand Frankreichs im Anhang der Forces productives et commerciales de la France; S.D.], gerade die Gebirgsgegenden es sind, die sich am meisten der Wohltaten des Schulunterrichts erfreuen; im Flachland ergibt sich als Verhältniszahl ein Achtel oder ein Zehntel; in Frankreich ist dies das Maximum. ${ }^{29}$

Z. 20f.: Strf. Ildegonde | Kreutzfahrer - Goethe und der Berliner Beamte, Schriftsteller und Übersetzer Adolph Friedrich Carl Streckfuß (1778-1844) standen seit 1824 in Verbindung und hatten sich im September 1827 in Weimar persönlich kennengelernt. Die auf Goethes Anfrage hin ${ }^{30}$ verfaßten Aufsätze Manzoni's Roman: Die Verlobten und Antonio Foscarini tragedia di G. B. Niccolini hatte ihm Streckfuß Mitte November zugeschickt. Sie wurden im 2. Heft des 6. Bandes von Ueber Kunst und Alterthum abgedruckt (FA I 22, S. 420-423 und 423426). In seinem Brief an Streckfuß vom 26. November 1827 (WA IV 43, S. 182-184) bedankte sich Goethe und übersandte ihm zugleich die beiden auf der Agenda vermerkten Werke des italienischen Dichters Tommaso Grossi (17901853): das 1820 erschienene Rittergedicht Ildegonda und das dreibändige Heldengedicht I Lombardi alla prima crociata (Die Lombarden auf der ersten Kreuzfahrt, 1826) mit der vorsichtigen Bitte um eine Rezension für das nächste Heft von Ueber Kunst und Alterthum. Ildegonda hatte bereits 1821 großes Interesse wie auch beträchtlichen Widerwillen bei Goethe ausgelöst ${ }^{31}$ - diese Ambivalenz zeigt sich auch im angeführten Brief an Streckfuß:

ich durfte es nicht zum zweytenmale lesen, weil es mir Einbildungskraft und Gefühl zu sehr verletzte. Dabey aber konnt ich ein bedeutendes poetisches Talent, Fähigkeit und Fertigkeit, Situationen der mannichfaltigsten Art kräftig darzustellen, nicht verkennen. (WA IV 43, S. 183)

Auf das epische Gedicht I Lombardi alla prima crociata, das 1843 als Vorlage für das Libretto zu Giuseppe Verdis gleichnamiger Oper diente, ist Goethe wohl über die Verbindungen Carl Augusts mit Gaetano Cattaneo aufmerksam gemacht geworden. ${ }^{32}$ Verstärkt wurde sein Interesse möglicherweise durch die intensive Lektüre von Alessandro Manzonis I promessi sposi (Die Verlobten, 1825/26) im Juli und August 1827. Im 11. Kapitel dieses Romans zitierte Man-

29 Frédéric Soret: Zehn Jahre bei Goethe. Erinnerungen an Weimars klassische Zeit. 1822-1832. Zusammengestellt und übers. von H. H. Houben. Leipzig 1929 (Reprint Hildesheim, Zürich und New York 1991), S. 215. Vgl. auch die Erwähnung von Dupins Karte in Eckermanns Gespräch mit Goethe, 23.10.1828 (FA II 12, S. 681f.).

30 Siehe Goethes Brief an Streckfuß, 27.10.1827 (WA IV 43, S. 135-137).

31 Siehe Tag- und Jahres-Hefte 1821 (FA I 17, S. 330f.).

32 Siehe Cattaneos Brief an Carl August vom 15. 11. 1825, in: Weimar und Mailand. Briefe und Dokumente zu einem Austausch um Goethe und Manzoni. Hrsg. von Hugo Blank. Heidelberg 1992, S. 271-273. 
zoni einen Vers aus Grossis Heldengedicht und weckte mit dem folgenen Kommentar die Neugierde seiner Leser:

Jener Vers stammt übrigens - wenn das jemand wissen möchte - aus einem unveröffentlichten, verteufelt guten Werk über Kreuzzüge und Lombarden, das schon bald nicht mehr unveröffentlicht sein wird und einiges Aufsehen erregen dürfte. ${ }^{33}$

In seinem Brief vom 31. Dezember 1827 urteilte Streckfuß über Grossis Gedichte, ,daß ein wahrhaft eminentes Talent zwey sehr schlechte und verwerfliche Werke geliefert hat. ${ }^{\text {‘34 }}$ Eine Rezension für Ueber Kunst und Alterthum kam nicht zustande.

Z. 23 li.: Schorn - In seinem Brief vom 27. Oktober 1827 hatte Goethe den sich in München aufhaltenden Kanzler Müller um Nachrichten für das 2. Heft des 6. Bandes von Ueber Kunst und Alterthum gebeten (WA IV 43, S. 130). Müller notierte in seinem Münchener Tagebuch unter dem 2. November 1827 einen Besuch bei Johann Karl Ludwig Schorn (1793-1842), Kunsthistoriker, Professor der Kunstgeschichte in München, seit 1820 Herausgeber des Kunstblattes zu Cottas Morgenblatt für gebildete Stände: „Gegen 6 Uhr bey Schorns. Correspondenz mit Göthe eingeleitet. ${ }^{\text {‘35 }}$ Als er Ende November von seiner Reise zurückkehrte, brachte Müller von Schorn für Goethe folgendes mit:

[...] acht Tafeln zu dem von Cotta übernommenen Werk Zahns „Neu entdeckte Wandgemälde in Pompeji in 40 Steinabdrücken“ [...], ferner mehrere Bildertafeln zu den gleichfalls bei Cotta erscheinenden „Antiken Bildwerken" von Friedr. Wilh. Eduard Gerhard [...]. (WA IV 44, S. 344)

Goethe vermerkte seine Beschäftigung mit den Münchner Sendungen im Tagebuch vom 28. - 30. November 1827 (WA III 11, S. 142f.). Beide genannten Werke wurden im 2. Heft des 6. Bandes von Ueber Kunst und Alterthum von Heinrich Meyer besprochen (FA I 22, S. 440f.).

Z. 23 re.: Unterschriften. Exp. fortgeführt - Die Eintragungen stehen möglicherweise im Zusammenhang mit folgenden Tagebuchnotizen Goethes: „Die Munda der Berichte unterschrieben“ (4. Dezember 1827, WA III 11, S. 144) und: „Vieles zu nächster Expedition vorgesprochen und vorbereitet.“ (5. Dezember 1827, WA III 11, S. 145) Aufgrund des allgemeinen Charakters der Notizen ist ihre Zuordung nicht mit Sicherheit möglich.

Z. 24 li.: Cornelius - In Goethes Tagebuch findet sich unter dem 1. Dezember 1827 der Eintrag: „Kamen die Kupfer zu Faust von Cornelius aus Frankfurt an.“ (WA III 11, S. 143.) Goethe kannte die Faust-Illustrationen von Peter Joseph Cornelius (1783-1867), Maler im Umkreis der Nazarener, seit 1825 Direktor der Kunstakademie in München, bereits seit Mai 1811. Sie erschienen 1816, gestochen von Ferdinand Ruscheweyh, in Frankfurt am Main. Schon im 2. Heft von Ueber Kunst und Alterthum (1817) waren sie in Heinrich Meyers von Goethe mitverantworteter Streitschrift Neu-deutsche religios-patriotische Kunst bespro-

${ }^{33}$ Alessandro Manzoni: Die Verlobten. Eine Mailändische Geschichte aus dem siebzehnten Jahrhundert. Übers. von Ernst Wiegand Junker. München 1989, S. 276. Vgl. Opere di Alessandro Manzoni. Hrsg. von Lanfranco Caretti. Mailand ${ }^{3} 1967$, S. 404. Manzonis Zitat aus I Lombardi alla prima crociata betrifft Vers 5 aus der 16. Strophe des 10. Gesanges (vgl. Tommaso Grossi: Opere poetiche. Hrsg. von Raffaele Sirri. Neapel 1972, S. 224).

${ }^{34}$ Weimar und Mailand (Anm. 32), S. 379.

${ }^{35}$ Von Müller (Anm. 15), S. 171. 
chen worden. ${ }^{36}$ In seinem Aufsatz Nauwerk, Bilder zu Faust im 1828 erschienenen 2. Heft des 6. Bandes seiner Kunstzeitschrift erwähnte sie Goethe zusammen mit den Faust-Illustrationen von Moritz Retzsch und Eugène Delacroix nochmals als „Zeugnisse einer ehrenvollen Theilname“ (FA I 22, S. 514). Gleichwohl äußerte sich Goethe im Gespräch mit dem Münchener Hofmaler Karl Joseph Stieler im Frühjahr 1828 kritisch über Cornelius' Illustrationen:

Vergebens war Stielers Bemühen, den Weimarer Olymp in seiner festgewurzelten Überzeugung zu erschüttern. Goethe kannte die neueren Leistungen [in der bildenden Kunst; S.D.] nur unvollkommen; für das aber, was er von ihnen kannte, besaß er das nötige Verständnis nicht; sonst wäre es unmöglich gewesen, dem Stielerschen Lobe des Faust von Cornelius das Bekenntnis entgegenzusetzen: „Ich mag darum (nämlich weil es ihm an der technischen Vollkommenheit fehle) Cornelius' Faust nicht leiden; es tritt nicht auseinander, er ist mir zu altdeutsch. $[\ldots]^{637}$

Z. 24 re.: Schütz Federn - Der Eintrag bezieht sich auf Schreibfedern, die Goethe dem Berkaer Organisten und Badeinspektor Johann Heinrich Friedrich Schütz (1779-1829) im Dezember 1827 zukommen ließ. Drei Tagebucheinträge Goethes stehen im direkten Zusammenhang mit der Agendanotiz: „Die drey Federn für Herrn Schütz ausgefertigt“ (4. Dezember 1827, WA III 11, S. 144), „Herrn Bade-Inspector Schütz die bescheinigten Federn abgesendet“" (5. Dezember 1827, WA III 11, S. 145) sowie „Inspector Schütz von Berka. Seine Freude über die wohlausgestatteten Federn bezeugend.“ (19. Dezember 1827, WA III 11, S. 150.) - Ende September 1827, also im unmittelbaren zeitlichen Kontext der Agenda, war Goethe mit Eckermann bei Schütz in Berka zu Gast gewesen. ${ }^{38}$ Vor allem als Kenner und Interpret der damals nahezu vergessenen Werke Johann Sebastian Bachs war Schütz, der auch Autographen des Komponisten besaß, für Goethe von großer Bedeutung. ${ }^{39}$

Z. 25 li.: Ringseisen - Der Eintrag steht aller Wahrscheinlichkeit nach im Zusammenhang mit Goethes weitreichender Beschäftigung mit der Mineralogie. Kanzler Müller traf den Medizinprofessor Johann Nepomuk von Ringseis (1785-1880) und seine Frau Friederike (1791-1877) während seines Aufenthalts in München am 25. Oktober und 8. November $1827 .^{40}$ Offenbar gab Ringseis Müller für Goethe Gesteinsproben mit, denn am 1. Dezember 1827 notierte dieser in seinem Tagebuch: „Die Münchner Mineralien besehen und beurtheilt.“ (WA III 11, S. 143) Am 4. Dezember 1827 übersandte sie Goethe dem Jenaer Bibliotheks- und Museumsschreiber Johann Michael Christoph Färber und schrieb im Begleitbrief dazu: „Hiebey erfolgt Abrazit vom Capo di Bove, zwey Stücke. Ein Schächtelchen mit Augiten, Beyde von Herrn von Ringseisen in München." (WA IV 51, S. 543.) - Die Augiten sollten dabei auf Goethes besonderes Interesse gestoßen sein, hatte er sich doch - zusammen mit dem Mineralogen Frédéric Soret - mit diesem zur Gruppe der Pyroxene gehörenden Silikat von Calcium, Magnesium und Eisen wiederholt, wie z.B. in der Studie Der Wolfsberg (1824) oder in dem (erst postum veröffentlichten) Aufsatz Von den Augiten insbesondere, beschäftigt.

\footnotetext{
36 Siehe FA I 20, S. 105-129, hier: S. 120.

37 Gespräche (Anm. 17), Bd. 3.2, S. 320, Nr. 6206.

38 Siehe Goethes Tagebuch, 24.9.1827 (WA III 11, S. 114).

39 Vgl. dazu Claus Canisius: Goethe und die Musik. München 1999, S. 110-114.

40 Siehe von Müller (Anm. 15), S. 170 und 172.
} 
Z. 25 re.: Boisserée Kästchen - Möglicherweise steht der Eintrag im Zusammenhang mit den Gipsabdrücken der Gemmensammlung des Baron Philipp von Stosch, geliefert in fünf Mahagonikästchen, die Goethe im Juli 1827 erworben hat. ${ }^{41} \mathrm{Er}$ erwähnte sie in seinem Brief an Sulpiz Boisserée (1783-1854) vom 11. November 1827:

Von Kunstwerken aquirirte ich bedeutende ältere Zeichnungen und verschaffte mir die Abdrücke der Stoschischen Sammlung, welche in Berlin sehr lobenswerth gefertigt und, nach dem alten Winckelmannischen Katalog geordnet, in zierlichen Kästchen ausgegeben werden [...]. (WA IV 43, S. 162)

Wohl im Februar 1828 verfaßte Goethe für das zweite Heft des sechsten Bandes von Ueber Kunst und Alterthum die kurze Besprechung Verzeichniß der geschnittenen Steine, die sich auf den im Brief genannten Winckelmannschen Katalog der Stoschischen Gemmensammlung bezieht. ${ }^{42}$

Z. 26 li.: Klenze - Auch dieser Eintrag auf der Agenda steht wohl im Zusammenhang mit der Münchenreise des Kanzlers Müller im Oktober/November 1827, über die er Goethe am 27. und 28. November Bericht erstattete (WA III 11, S. 142). Wie Schorn (vgl. Z. 23 1.), Cornelius (vgl. Z. 24 1.) und Ringseis (vgl. Z. 25 1.) hatte Kanzler Müller während seines Aufenthalts in München auch den Architekten, Archäologen, Maler und Münchener Oberbaudirektor Franz Leopold (Leo) Karl von Klenze (1784-1864) besucht. In seinem Tagebuch notierte er unter dem 28. Oktober 1827: „Bey Klenze [...] Goethes Worte: ,Was ist die Baukunst anderes, als eine zweite Natur, den menschlichen Bedürfnissen angeeignet', haben Klenzen einst neuen Schwung und Richtung gegeben." ${ }^{\text {43 }}$ Im April 1828 übersandte Klenze Goethe sein Ölgemälde vom Tempel des Jupiters von Girgenti, das im 2. Heft des 6. Bandes von Ueber Kunst und Alterthum von Goethe unter dem Titel Südöstliche Ecke des Jupiter-Tempels von Girgent sehr positiv besprochen wurde (FA I 22, S. 499f.).

Z. 26 re.: Reichel Nachr. - Vgl. den Kommentar zu Z. 6. Die Notiz bezieht sich wohl auf Goethes Brief an Reichel vom 7. Dezember 1827 (WA IV 43, S. 201), in dem er den Empfang der zweiten Lieferung der Ausgabe letzter Hand bestätigte und Korrekturen zum 5. Band (Gedicht-Teil des West-östlichen Divans) übersandte.

Z. 27 li.: Nürnbg Subs. - Der Eintrag steht wohl im Zusammenhang mit dem geplanten Dürer-Denkmal in Nürnberg, für das Goethe Subskribenten anwerben sollte. Anläßlich des 300. Todestags Albrecht Dürers plante man in seiner Vaterstadt Nürnberg die Errichtung eines Denkmals. Daß tatsächlich ein ,Erinnerungsbedarf' bestand, wird in Zelters Nürnberger Brief an Goethe vom 7. Oktober 1827 deutlich:

Es ist einmal so; bei aller Bewundrung der Nachwelt haben es die großen Werke nicht besser als ihre Schöpfer. Wer es nicht um Seinselbst willen täte dürfte wenig Freude haben, am Egoismus der Andern. Von 5-6 Einwohnern [Nürnbergs] wußte mir kaum Einer Dürers Leichenstein nachzuweisen, den ich mir selbst fand, welches unter einigen Tausend Steinen kein leichtes ist. (MA 20.1, S. 1058f.)

\footnotetext{
41 Siehe Goethe's Kunstsammlungen [T. 3: Sammlungen]. Beschrieben von Chr[istian] Schuchardt u. A. Jena 1848f., T. 2, S. 344, Nr. 270.

42 Siehe FA I 22, S. 507f., und den Kommentar.

43 Von Müller (Anm. 15), S. 171.
} 
Der Nürnberger Auktionator, Buch- und Kunsthändler Johann Lorenz Schmidmer hatte Goethe am 17. Dezember 1827 gebeten, Subskribenten für das Dürer-Denkmal anzuwerben, ${ }^{44}$ dessen Ausführung der Berliner Bildhauer Christian Daniel Rauch übernommen hatte. In seinem Brief an Kanzler Müller vom 7. November 1827 skizzierte Goethe die Zusammenhänge und machte deutlich, daß er sich nicht an der Organisation der Subskription beteiligen würde:

In Nürnberg können Sie mir einen großen Dienst erzeigen; Herr Schmidmer, sich auf sein altes gutes Verhältniß beziehend, schickt mir ein großes Paquet zu vertheilender und verschickender Subscriptions-Anzeigen; die guten Menschen bedenken nicht, daß ich in meinem hohen Jahren mich mit einem so complicirten Geschäft nicht befassen, eine weitläufige Correspondenz deshalb nicht führen kann. (WA IV 43, S. 152)

Gleichzeitig sicherte Goethe jedoch publizistische Unterstützung für das Projekt zu. Im Briefwechsel zwischen Goethe und Rauch ist in den folgenden Monaten wiederholt vom Dürer-Denkmal die Rede. ${ }^{45}$ Die Grundsteinlegung für das Denkmal erfolgte am 6. April 1828. Über die Fortschritte des erst 1840 enthüllten Dürer-Denkmals konnte Goethe aber nicht mehr öffentlich berichten.

Z. 27 re.: Lechner Nachr. Zahlung - Am 6. November 1827 hatte Goethe bei dem Nürnberger Auktionator und Kunsthändler Johann Jacob Lechner zwei Kisten mit Majolika bestellt (WA IV 43, S. 150f.). Am 3. Dezember 1827 traf die Sendung ein: „Die von Nürnberg angekommenen Majolikas ausgepackt, betrachtet und beurtheilt.“ (WA III 11, S. 144) In seinem Brief an Lechner vom 4. Dezember 1827 bestätigte Goethe den Empfang und unterrichtete ihn von seiner Zahlungsanweisung: ,[...] vermelde daß gestern, den 3. December, die übersendete Majolika glücklich angekommen ist. [...] Herr Banquier Elkan erhält so eben den Auftrag, Ihnen 131 Thaler sächsisch für gedachte Sendung auszuzahlen [...].“(WA IV 43, S. 197f.)

Z. 28: Elkan Lechner - Der Eintrag bezieht sich auf die Zahlungsanweisung Goethes für die Majolika von Lechner im Brief an seinen Bankier Julius Elkan (1781-1839) vom 4. Dezember 1827 (WA IV 43, S. 198). Vgl. den Kommentar zu Z. 27 re.

Z. 29: Nicolovius - Die Notiz bezieht sich wahrscheinlich auf Alfred Nicolovius (18061890), Jurist, Großneffe Goethes, Enkel seiner Schwester Cornelia, oder möglicherweise auf dessen Bruder Heinrich (1798-1868), Jurist.

Alfred Nicolovius, der vom 31. Oktober bis 3. November 1827, also im zeitlichen Kontext der Agenda, auch in Weimar zu Gast war, schickte am 25. November 1827 aus Berlin eine umfangreiche Sendung von Kunstgegenständen, Aufsätzen u.a. an Goethe ab. In seinem Brief vom 4. Dezember 1827 wies Goethe den Bankier Elkan (vgl. Z. 28) an, Nicolovius seine Auslagen zu erstatten (WA IV 43, S. 198). Einige Stücke dieser Sendung wurden im 2. Heft des 6. Bandes von Ueber Kunst und Alterthum von Goethe besprochen, der sich dabei teilweise auch auf Notizen von Alfred Nicolovius stützte. ${ }^{46}$

Sein Bruder Heinrich hielt sich im Dezember 1827 zweimal für einige Tage in Weimar auf. Vom 2. - 6. sowie vom 22. - 26. Dezember wird sein Besuch in Goethes Tagebuch wiederholt vermerkt (WA III 11, S. 143-145 und 152-154).

\footnotetext{
44 Siehe WA IV 43, S. 365.

45 Siehe z.B. Rauchs Brief an Goethe vom 18. 1. 1828 (Karl Eggers: Rauch und Goethe. Urkundliche Mittheilungen. Berlin 1889, S. 178).

46 Vgl. z.B. den Aufsatz Granitarbeiten in Berlin (FA I 22, S. 508).
} 
Z. 30: Cäcilia Fr. v. Pogwisch - Der Agendaeintrag bezieht sich wohl auf Cäcilia. Zeitschrift für die musikalische Welt, Mainz 1814-1829, sowie auf Henriette Ottilie Ulrike Freifrau von Pogwisch (1776-1851), seit 1809 Hofdame der Herzogin Louise am Hof zu Weimar, Mutter von Goethes Schwiegertochter Ottilie. Eine Verbindung zwischen den Einträgen „Cäcilia“ und „Fr. v. Pogwisch“ konnte nicht ermittelt werden.

Z. 31:Buchb. Bauer. 2. Lieferung - Die Notiz bezieht sich auf einen Auftrag Goethes an den Weimarer Buchbinder Bauer. In Goethes Tagebuch findet sich am 4. Dezember 1827 der folgende Eintrag: „Die Exemplare der zweyten Lieferung [der Ausgabe letzter Hand seiner Werke; S.D.] ausgepackt und an die verschiedenen Interessenten vertheilt. Auch Bauern 14 Exemplare zum Heften gegeben.“ (WA III 11, S. 144)

Z. 32: Zelter nach dem Concept - Gemeint ist wohl Goethes Brief an Carl Friedrich Zelter (1758-1832) vom 4. Dezember 1827, zu dem ein Konzept von Johann Christian Schuchardts Hand vorliegt. ${ }^{47}$ Goethe äußert sich in diesem Schreiben unter anderem über seine Lektüre von Walter Scotts 1827 erschienenem Werk The Life of Napoleon Buonaparte. ${ }^{48}$

Z. 33: B. Cotta. Schiller - Der Eintrag bezieht sich auf den Brief Goethes vom 17. Dezember 1827 an Johann Friedrich Cotta (1764-1832), der den Briefwechsel zwischen Schiller und Goethe zum Gegenstand hat. Seit 1822 trug sich Goethe mit Plänen zur Herausgabe dieser Korrespondenz. Der Teilabdruck in Ueber Kunst und Alterthum (Bd. 5, H. 1 und 2 sowie Bd. 6, H. 1) erfolgte bereits im Hinblick auf die geplante Gesamtausgabe, die 1828/29 erschien. Vorausgegangen waren - unter Vermittlung Sulpiz Boisserées - schwierige Verhandlungen zwischen Herausgeber und Verleger, nicht zuletzt wegen der beträchtlichen finanziellen Forderungen Goethes. In dem angeführten Brief machte Goethe die Übersendung des Manuskripts von der vorherigen Überweisung einer Summe von 8000 Talern abhängig, erst dann käme das „Geschäft, das mir viele Mühe, Sorgen und Kosten gemacht, [...] doch endlich zu Stande.“ (WA IV 43, S. 209) Cotta reagierte in seinem Antwortschreiben vom 11. Februar 1828 äußerst gekränkt und bezeichnete Goethes Brief als „Schlußstein eines sehr kummervollen Jahres“ ${ }^{49}$ Erst ein halbes Jahr später nahmen beide wieder brieflichen Kontakt miteinander auf.

Z. 34: Jouy. Royer Collard - Vgl. den Kommentar zu Z. 12 re.

Z. 35: Dr. Levi - Vgl. den Kommentar zu Z. 13 re.

Z. 36: Rechnungen. Horny, Hofmann - Diese Notiz läßt sich nicht mit Sicherheit zuordnen. Möglicherweise steht sie im Zusammenhang mit Goethes amtlicher Tätigkeit und bezieht sich auf das Weimarer Geschäft Horny bzw. seine Inhaberin, Josephe Horny, sowie auf den Kammerbeamten und Weimarer Kassenkontrolleur Carl Friedrich Hoffmann (bzw. Hofmann).

Über das Geschäft von Josephe Horny, in dem Goethe auch für seinen eigenen Bedarf Papier kaufte, ${ }^{50}$ bezog der Weimarer Hof Schreibmaterialien für seine Beamten. In seiner Funktion als Minister mit „Oberaufsicht über die unmittelbaren Anstalten für Wissenschaften und Kunst in Weimar und Jena" ließ Goethe Josephe Horny am 29. März 1828 eine Verfügung zukommen, „künf-

47 Siehe WA IV 43, S. 194-197 und 388.

48 Vgl. Goethes Rezensionsentwurf vom 20./21.11.1827 für Ueber Kunst und Alterthum (Walter Scot. Leben Napoleons, FA I 22, S. 706-708).

49 Briefe an Goethe. Hamburger Ausgabe in zwei Bänden. Gesammelt, textkritisch durchgesehen und mit Anmerkungen versehen von Karl Robert Mandelkow. Hamburg 1965-1969, Bd. 2, S. 472.

50 Siehe Goethes Briefe an Kräuter vom 15. und 21. Oktober 1821 (WA IV 35, S. 143 u. 149f.). 
tighin nur auf Bescheinigung von mir selbst es sey was es wolle aus ihrem Laden abzugeben, auch diese den vierteljährig einzusendenden Rechnungen jederzeit beyzulegen, weil sonsten die Bezahlung nicht erfolgen kann." (WA 44, S. 348)

Carl Friedrich Hoffmann war für die Buchführung im Zusammenhang mit Goethes amtlicher Tätigkeit zuständig. ${ }^{51}$ Sein Name findet sich im letzten Lebensabschnitt häufig in den Tagebucheinträgen und auf Agenden. ${ }^{52} \mathrm{Im}$ vorliegenden Zusammenhang könnte eine Notiz vom 8. Januar 1828 einschlägig sein: „An den Controleur Hoffmann hier.“ (WA III 11, S. 161) Der entsprechende Brief Goethes ist jedoch nicht überliefert.

Als weitere Bezugspersonen für die Agendanotiz „Hofmann“ kommen in Frage: der Buchhändler Johann Wilhelm Hoffmann ${ }^{53}$ sowie der Hofapotheker Karl August Hoffmann (1755 od. 1756-1833) ${ }^{54}$. Mit beiden Weimarer Persönlichkeiten stand Goethe im geschäftlichen Kontakt.

Z. 37: Canzl. Gedicht - Der Eintrag bezieht sich auf Friedrich Theodor Adam Heinrich von Müllers (1779-1849, seit 1815 Kanzler, d.h. Justizminister in Weimar) Gedicht Dem Könige die Muse. 28. August 1827. Dieses im September 1827 auf Anregung Goethes entstandene und entsprechend seinen Anmerkungen überarbeitete 32-strophige „Gelegenheitsgedicht“ ist ein poetischer Dankesbeweis Weimars für den Besuch des Bayerischen Königs Ludwig I. zu Goethes Geburtstag am 28. August 1827. Kanzler Müller hatte es während seines Aufenthalts in München am 27. Oktober 1827 Ludwig übergeben ${ }^{55}$ und auch auf einigen seiner sonstigen Münchener Besuche vorgelegt. In seinem Brief an Goethe vom 1. November 1827 schilderte Müller die Reaktion Schellings:

Er gehört zu dem kleinen Kreise, dem ich mein Gedicht mittheilte, und er gab mir zu verstehen, daß eine Veröffentlichung deßelben wohl im Sinne des Königs seyn möchte. Da entspann sich denn der weitere Gedanke, ob Sie ihm nicht eine Stelle in Kunst und Alterthum gönnen wollten, indem der König darinn seines Besuchs erwähnt zu sehen, wohl ohnehin gewärtig seyn dürfte und es nicht fehlen würde, daß in dem goldnen Rahmen Ihrer Einfaßung auch das minder Bedeutende sich Gunst gewönne. ${ }^{56}$

${ }^{51}$ Nach freundlicher Auskunft von Dr. Elke Richter, Stiftung Weimarer Klassik (GoetheBriefrepertorium).

52 Siehe die Tagebucheinträge vom 11. April 1827 sowie vom 1. und 2. Januar 1831 (WA III 11, S. 44 und WA III 13, S. 1f.), die Notizen auf den Agenden vom 14. Dezember 1830, vom Januar 1831, vom Mai 1831, vom Juni 1831 sowie vom Juli/August 1831 (WA III 13, S. 260, 262, 269, 270 und 271) sowie das bisher unpublizierte Schreiben Goethes an Carl Friedrich Hofmann vom 26. Juni 1828 (WA-Nr. 44137a+. In: Johann Wolfgang Goethe: Repertorium sämtlicher Briefe 1764-1832. Hrsg. von der Stiftung Weimarer Klassik/Goethe- und Schiller-Archiv. Begründet von Paul Raabe an der Herzog August Bibliothek Wolfenbüttel. Bearbeitet von Elke Richter unter Mitarbeit von Andrea Ehlert, Susanne Fenske, Eike Küstner und Katharina Mittendorf. URL: http://ora-web.weimar-

klassik.de/goethe_2_online/repertorium.vollanzeige1?d_id=015453 [26. August 2001]).

53 Siehe Goethes Brief an Johann Wilhelm Hoffmann vom 28. Dezember 1827 (WA IV 43, S. 213), in dem es allerdings nicht um eine Rechnungsangelegenheit geht.

54 Siehe Karl August Hoffmann an Goethe, 27.10.1827 (GSA 28/125 B1. 524). Auch hier geht es nicht um eine Rechnungsangelegenheit. (Nach freundlicher Auskunft von Frau Sabine Schäfer, Stiftung Weimarer Klassik/Goethe- und Schiller-Archiv.)

55 Siehe von Müller (Anm. 15), S. 170.

56 Von Müller (Anm. 15), S. 342. 
Tatsächlich entschied sich Goethe in der ersten Dezemberhälfte, mit Müllers Gedicht das nächste Heft (Bd. 6, H. 2) von Ueber Kunst und Alterthum zu eröffnen (FA I 22, S. 403-411). Doch Müllers/Schellings Idee vom ,goldnen Rahmen' einer Goetheschen 'Einfassung' des Gedichts fand eine noch weiter reichende Umsetzung: Bereits am 6. Januar 1828 wurden laut Goethes Tagebuch die „Noten zu dem von Müllerischen Gedicht besprochen“ (WA III 11, S. 160), Kommentare Goethes Zum nähern Verständniß des Gedichts: „Dem Könige die Muse." gewidmet dem 28. Aug. 1827, ebenfalls abgedruckt im 2. Heft des 6. Bandes von Ueber Kunst und Alterthum (FA I 22, S. 470-473).

Z. 38: Bergr. Kramer - Ludwig Wilhelm Cramer (1755-1832), Mineraloge, Oberbergrat in Wiesbaden, später in Dillenburg und Wetzlar, den Goethe von seinen Wiesbadener Aufenthalten 1814 und 1815 kannte, hatte diesem am 27. November 1827 seine Geognostischen Fragmente von Dillenburg und der umliegenden Gegend $^{57}$, das letzte Exemplar Goethit (Nadeleisenerz, 1806 zu Goethes Ehren umbenannt) aus seiner Sammlung und weitere mineralogische Raritäten zugesandt. ${ }^{58}$ Goethe bedankte sich dafür in seinem Brief an Cramer vom 4. Januar 1828 (WA IV 43, S. 228f.).

Z. 39: das Neuste von Pluw. - Der Eintrag bezieht sich auf Goethes Gedicht Das Neueste von Plundersweilern (1781) und die dazugehörige Zeichnung von Georg Melchior Kraus (1737-1806). Gedicht und Bild sind Gegenstand zweier Tagebucheinträge Goethes vom Dezember 1827: „Hofrath Meyer hatte die Zeichnung, ,das Neuste von Plundersweilern' geschickt“ (4. Dezember 1827, WA III 11, S. 144) und: „Das Neuste von Plundersweilern an Hofrath Meyer.“ (6. Dezember 1827, WA III 11, S. 145) Für den Abdruck der Literatursatire und der Zeichnung in der Ausgabe letzter Hand verfaßte Goethe am 6. Dezember 1827 eine neue Einleitung, in der die Entstehungsgeschichte referiert wird (FA I 5, S. 1105).

57 Ruppert (Anm. 20), S. 642, Nr. 4471.

58 Siehe WA IV 43, S. 406. 